Neurological Impairment (SNI) in the literature. We aimed to develop an international, consensus-based, multi-disciplinary definition of this term.

Methods The Delphi process was chosen to achieve consensus on the definition of SNI. We collaborated with experts in 5 countries (Ireland, the UK, the USA, Canada and Australia) to disseminate an invitation to other colleagues in neurodisability in their own region. We specified that a multi-disciplinary panel was required. Those who wished to participate were asked to email us, as facilitators of the process, to confirm their desire to take part. Participants were asked to further disseminate the invitation to other colleagues, thus employing a snow-balling effect in the recruitment of expert panellists.

The Delphi process proceeded over 3 rounds. Round 1 used free-text responses where panellists provided insight into their understanding of the term SNI. Responses were used to generate themes. In rounds 2 and 3 panellists were asked to rate their agreement with these themes in the definition of SNI. In the process of round 3 participants were provided with feedback on the previous round, including anonymous information on how the other panellists had voted as well as selected written feedback to provide an opportunity to consider other points of view. Items were brought forward to the final definition if they received more than $70 \%$ agreement, in line with accepted Delphi methodology. After round 3, a working definition of SNI was created. Further refinements were made based on comments from parent representatives and experts at an international conference.

Results Thirty-four multi-disciplinary panellists participated in round 1 of the process falling to 31 in round 3, a 9\% dropout rate. Fifteen themes were generated from responses in round 1 . Seven items were brought forward for inclusion in the final definition.

Conclusion We have created an international, multi-disciplinary, consensus-based definition of SNI. This definition can be used to improve consistency in reporting of research, ultimately leading to improved outcomes for this unique and vulnerable cohort of children.

\section{G46(P) PROGRESSION OF NEUROPHYSIOLOGICAL CHANGES IN A CHILD WITH SUBACUTE SCLEROSING PANENCEPHALITIS}

${ }^{1}$ A Lazenbury, ${ }^{1} \mathrm{~N}$ Bhangu, ${ }^{1} \mathrm{M}$ Illingworth, ${ }^{2} \mathrm{D}$ Allen, IJ Singh. ${ }^{1}$ Department of Paediatric Neurology, University Hospital Southampton, Southampton, UK; ${ }^{2}$ Department of Clinical Neurophysiology, University Hospital Southampton, Southampton, UK

10.1136/archdischild-2020-rcpch.33

Subacute sclerosing panencephalitis (SSPE) is classically associated with characteristic electroencephalogram (EEG) features of periodic and stereotyped high voltage discharges. We present the electroclinical evolution in a case of SSPE in whom the typical changes were not initially evident. The patient is a fully immunized seven-year old boy with a sixmonth history of increasingly frequent atonic drops with behavioural change and cognitive decline. His initial EEG showed continuous high amplitude 1-2 $\mathrm{Hz}$ sharp and slow wave activity, which were frontally dominant with little change on midazolam infusion. Eight days later, the EEG had a diffusely slow background with anterior sharp waves in a periodic/semi-periodic fashion, with complexes every 1-2 seconds.
A rapid neurocognitive decline ensued, characterized by fluctuating consciousness, visual impairment, aphasia and the emergence of dystonia, rendering him non-ambulant within weeks of hospital admission. The EEG evolved with a diffusely slow background with no periodic sharp waves, except occasionally in the temporal regions. MRI brain showed asymmetrical T2-weighted signal change in sub-cortical and deep white matter. Serial imaging demonstrated increasing widespread signal abnormality. SSPE was confirmed by CSF measles PCR. His EEG at ten weeks after presentation progressed to typical periodic sharp and slow wave complexes, occurring every 6-7 seconds.

The clinical course and EEG add weight to the growing body of evidence of SSPE occurring in a younger than expected cohort across the UK and Europe. SSPE is a neurodegenerative disorder where early illness is known to have a higher association with development of measles related sequelae. At a time when vaccination uptake is at an all time low, increased awareness of the burden of measles is crucial to facilitate improved health promotion and vaccination uptake worldwide. SSPE should be considered in any child presenting with regression and seizures even if the EEG and neuroimaging are not initially wholly supportive.

\section{G48(P) WRIST-WORN DEVICES TO IMPROVE ARM MOVEMENT IN HEMIPLEGIC CEREBRAL PALSY: PARTICIPATORY DESIGN WORKSHOPS}

${ }^{1} \mathrm{R}$ Brown, ${ }^{2} \mathrm{~J}$ Pearse, ${ }^{3} \mathrm{G}$ Edmonds, ${ }^{4} \mathrm{~T}$ Nappey, ${ }^{4} \mathrm{~K}$ Ladha, ${ }^{4} \mathrm{D}$ Jackson, ${ }^{4} \mathrm{Y}$ Guan, ${ }^{3,5} \mathrm{~A}$ Basu. ${ }^{1}$ Psychology, Newcastle University, Newcastle upon Tyne, UK; ${ }^{2}$ Occupational Therapy, Great North Childrens Hospital, Newcastle upon Tyne, UK; ${ }^{3}$ Institute of Health and Society, Newcastle University, Newcastle upon Tyne, UK; ${ }^{4}$ Computing Science, Newcastle University, Newcastle upon Tyne, UK; ${ }^{5}$ Paediatric Neurology, Great North Childrens Hospital, Newcastle upon Tyne, UK

\subsection{6/archdischild-2020-rcpch.34}

Background Hemiplegic Cerebral Palsy causes unilateral weakness and stiffness. Upper limb therapy at effective intensity is not accessible to most.

Aim Determine stakeholder views on design of an approach using wrist-worn devices and software (phone application) incorporating positive feedback and peer support, to encourage use of the affected arm and hand.

Method Five participatory design workshops, incorporating the views of 5 young people (YP) with hemiplegia and 13 typically developing (TD) (8-18 years) plus three parents, six researchers, three occupational therapists, one teacher and two paediatricians.

Results Four themes were noted: Ease/practical issues, Appearance/comfort, Incentives, and Access to participants. Ease/practical issues: Distinguishing between left and right devices proved challenging, so images of differing orientation were added. Concern about teachers' disapproval was resolved by producing an explanatory 'business card' for YP to hand out. Mobile phones are not required in school as the device and app can synchronise later, via Bluetooth. Concerns were addressed, regarding whether devices could gather secure information from arm movement, e.g. PIN numbers. Reassuringly, data is averaged over a minute, rendering this impossible. A parent asked whether GPs could use the data for other purposes (e.g. investigation of tremor) - but this would present challenges. Incentives: Games were a popular incentive, especially those with a competitive element. Inclusion of 
the game and summarised data showing progress was favoured. Parents were incentivised by this opportunity for their child to receive help and take responsibility for therapy. Appearance/comfort: The devices were programmed to display the time, in response to requests from YP. Wearing two devices was mildly perturbing for older participants as this would be considered unusual, though all were prepared to do it for the project duration. Access to participants: Recruitment to the workshops through advertising and patient groups was challenging; however, in the planned study, we will recruit through the NHS.

Conclusion The participatory design process informed plans for the proof of concept stage of the study, hopefully leading to a product and approach that will be fun, easy to integrate into everyday life, and have the capacity to increase use of the affected arm and hand.

\section{G49(P) RCPCH STROKE IN CHILDHOOD GUIDELINES: ARE THESE BEING FOLLOWED?}

YC Collins-Sawaragi, H Walker, J Sanperaiglesias, S Byrne, E Wraige, D Lumsden, S Tang. Paediatric Neurology, Evelina London Children's Hospital, London, UK

\subsection{6/archdischild-2020-rcpch.35}

Aims We investigate if the RCPCH Stroke in Childhood Guidelines (2017) are being followed in paediatric patients presenting with a 'brain attack'.

Methods We retrospectively collected two cohorts of paediatric patients aged over 2 years old presenting with symptoms suggestive of stroke ('brain attack') over 6 months between March and August 2019. Cohort 1 were patients from a Tertiary Hospital A\&E department that were identified through the Symphony electronic database. Cohort 2 were from patient referrals to the Tertiary Paediatric Neurology Department documented in the electronic database. Patient demographics, symptoms, NIHSS (National Institute of Health Stroke Scale), investigations, time to brain scan, thrombolysis and thrombectomy rates and final diagnosis were collected.

Results 33 patients in Cohort 1 and 32 patients in Cohort 2 had symptoms that met possible stroke diagnosis. Mean age of all patients were 8.9 years (36 male, 29 female). The top 3 presenting symptoms were focal seizures (20/65), speech disturbance $(14 / 65)$ and headache (13/65). Only 6 patients had NIHSS calculated. Cranial imaging was performed within 1 hour of admission in only $1 / 33$ in the A\&E cohort and 2/32 in the external referral cohort. $9 / 65$ patients had a diagnosis of stroke: 2 transient ischaemic attacks, 5 ischaemic strokes, 1 venous stroke and 1 haemorrhagic stroke. 28/65 patients had other diagnoses requiring urgent treatment such as seizure disorders and infection. In total 2 patients were evaluated for thrombolysis - 1 patient received thrombolysis under the care of the adult stroke team. 2 patients were referred for thrombectomy but none were suitable candidates (1 symptom resolution before thrombectomy, 1 high risk of complications due to anticoagulation post-operatively).

Conclusion RCPCH Stroke in Childhood Guidelines are not consistently being followed, particularly with regards to calculation of NIHSS and delays in obtaining brain imaging. If guidelines are not followed, paediatric stroke patients and windows of treatment with thrombolysis/thrombectomy may be missed. This highlights the importance of having a paediatric stroke pathway in place.

\section{G50(P) GETTING TO THE HEART OF THE WHITE MATTER}

${ }^{1}$ I Morgan, ${ }^{1} \mathrm{C}$ Herbert, ${ }^{2} \mathrm{~K}$ Forbes, ${ }^{3} \mathrm{M}$ O'Regan, ${ }^{4} \mathrm{~L}$ Hunter, ${ }^{1} \mathrm{~K}$ Forrest. ${ }^{1}$ Paediatric Neurology, RHC, Glasgow, UK; ${ }^{2}$ Paediatric Radiology, RHC, Glasgow, UK; ${ }^{3}$ Paediatric Neurology, Our Lady's Children's Hospital, Dublin, Ireland; ${ }^{4}$ Paediatric Cardiology, RHC, Glasgow, UK

\subsection{6/archdischild-2020-rcpch.36}

Objective Acute Disseminated Encephalomyelitis (ADEM) is an immune mediated inflammatory CNS disorder, predominantly affecting white matter, with a wide differential. ${ }^{1}$ Here we describe a rare mimic of ADEM that is essential to consider in order to avoid catastrophic outcome.

Case History A 12-year old girl presented with a 2 day history of confusion, dysarthria, ataxia and left-sided squint, preceded by 12 days of being generally unwell, with headache and lethargy. Examination confirmed encephalopathy and left third cranial nerve palsy.

MRI brain was suggestive of ADEM and MRI spine was normal. A recommended work up for $\mathrm{ADEM}^{1}$ was performed. Rapid resolution of her symptoms occurred following treatment with intravenous methylprednisolone for three days.

2 years later she presented with acute left lower limb ischaemia and underwent emergency embolectomy of a popliteal arterial obstruction; with myxomatous material identified. Preoperative echocardiogram confirmed left atrial mass which was then surgically removed. Pathology confirmed an atrial myxoma (AM).

Retrospective review of her initial MRI images concluded that embolic phenomena from the AM was the most likely explanation of her first presentation.

Conclusions AM is a very rare primary cardiac tumour and left sided AM can embolise to the cerebrovascular system. $12 \%$ of adults with AM present with neurological symptoms, and this can mimic multiple sclerosis. AM presenting with acute neurological symptoms masquerading as ADEM in paediatrics has not previously been reported. Early identification of $\mathrm{AM}$ is important as, untreated, it can cause multiple embolic events and sudden cardiac death. Careful follow up is essential as late neurological complications (including cerebral arterial aneurysms) are recognised.

This case highlights that ADEM is a diagnosis of exclusion, and that mimics for acute focal neurology with encephalopathy and T2 hyperintensities on MRI require careful consideration, including embolic phenomena. Clinical examination alone does not exclude AM and consideration of echocardiography is recommended.

\section{REFERENCE}

1. Differential diagnosis and evaluation in paediatric inflammatory demyelinating disorders. Neurology 2016 (Supp|2);87:S28-37.

\section{G51(P) INTERACTION OF CANNABIDIOL (CBD) WITH OTHER ANTISEIZURE MEDICATIONS (ASMS)}

${ }^{1}$ CGS Gilmartin, ${ }^{2} Z$ Dowd, 2 PD Harijan. 'School of Clinical Medicine, University of Cambridge, Cambridge, UK; ${ }^{2}$ Department of Paediatric Neurology, Cambridge University Hospitals NHS Trust, Cambridge, UK

\subsection{6/archdischild-2020-rcpch.37}

Objective Cannabidiol is efficacious as an adjunctive treatment in children with epilepsy associated with Dravet and LennoxGastaut syndromes. As cannabidiol is currently co-prescribed with other antiseizure medications (ASMs), a literature review 